\title{
Disparate Mg II Absorption Statistics Towards Quasars and Gamma-Ray Bursts: \\ A Possible Explanation
}

\author{
S. Frank ${ }^{1}$, M. C. Bentz ${ }^{1}$, K. Z. Stanek ${ }^{1}$, S. Mathur ${ }^{1}$, M. Dietrich ${ }^{1}$, B. M. Peterson ${ }^{1}$, \\ D. W. Atlee ${ }^{1}$ \\ frank@astronomy . ohio-state.edu
}

\begin{abstract}
We examine the recent report by Prochter et al. that gamma-ray burst (GRB) sight lines have a much higher incidence of strong Mg II absorption than quasar sight lines. We propose that the discrepancy is due to the different beam sizes of GRBs and quasars, and that the intervening Mg II systems are clumpy with the dense part of each cloudlet of a similar size as the quasars, i.e. $\lesssim 10^{16} \mathrm{~cm}$, but bigger than GRBs. We also discuss observational predictions of our proposed model. Most notably, in some cases the intervening Mg II absorbers in GRB spectra should be seen varying, and quasars with smaller sizes should show an increased rate of strong $\mathrm{Mg}$ II absorbers. In fact, our prediction of variable Mg II lines in the GRB spectra has been now confirmed by Hao et al., who observed intervening Fe II and $\mathrm{Mg}$ II lines at $z=1.48$ to be strongly variable in the multi-epoch spectra of $z=4.05$ GRB 060206 .
\end{abstract}

Subject headings: gamma-rays: bursts — quasars: absorption lines

\section{Introduction}

As some of the most luminous objects in the Universe, gamma-ray bursts (GRBs) have been established as important cosmological probes. In particular, the transient optical afterglows of GRBs are important for absorption line studies along lines of sight that are not associated with quasars (cf. Vreeswijk et al. 2003; Chen et al. 2005).

Recently, Prochter et al. (2006, hereafter P06) reported statistically significant evidence for a higher incidence of strong Mg II $\lambda \lambda 2796,2803$ absorbers towards GRB sight lines than

\footnotetext{
${ }^{1}$ Department of Astronomy, The Ohio State University, 140 W. 18th Ave, Columbus, OH 43210
} 
towards quasars of comparable redshifts. More precisely, they found 15 strong (rest frame equivalent width $\left.W_{\mathrm{r}}>1.0 \AA\right) \mathrm{Mg}$ II absorbing systems in a sample of 12 GRBs with optical follow-up spectroscopy allowing for both Mg II doublet components to be identified at $>3 \sigma$ confidence level. Based on the study of a Sloan Digital Sky Survey (SDSS) quasar sample with over 50,000 quasars containing more than 7,000 Mg II systems with $W_{\mathrm{r}}>1.0 \AA$, P06 expect only 3.4 such systems in quasar spectra over the redshift path interval of the GRB sample. Thus, about four times as many strong Mg II absorbers are observed along the line of sight to GRBs compared to quasar sight lines.

P06 propose and quickly dismiss three effects to explain the discrepancy in the observed and expected incidence of Mg II absorbers along GRB sight lines. First, they suggest that dust associated with the Mg II absorbers could obscure faint quasars resulting in a lower number of observations of such systems along quasar sight lines. Secondly, the absorbing gas could simply be associated with the GRB event, which they dismiss as unphysical because of the relativistic speeds necessary and the intrinsically narrow absorption line widths. As a third possibility, P06 propose that the GRB population could be gravitationally lensed by these absorbers; this does not seem to be the case as the lensing magnification necessary would be evident by bright foreground galaxies or multiple images of the source. P06 therefore conclude that "at least one of the fundamental beliefs on absorption line research must be flawed."

In this Letter, we propose a geometric solution for the observed difference in incidences and explore observational signatures of the proposed solution.

\section{Geometric Differences Between GRBs and Quasars}

Given that the P06 result is not affected by selection effects, gravitational lensing or Mg II absorbers local to GRB hosts, we are left with the scenario that GRB sight lines trace the same intervening absorber population as quasars. These intervening Mg II absorbers thus "know" nothing about the background light sources, and yet their number densities are different in GRB and quasar sightlines. Here we suggest a simple geometric explanation for the different number of strong Mg II absorbers per unit redshift along sight lines towards quasars and GRBs. We postulate that (1) the GRB sizes are, on average, smaller than quasar sizes, and (2) the Mg II absorbers are clumpy (or have density structure), with characteristic sizes on the order of GRB beam sizes. These simple assumptions lead to the observed differences due to dilution of the Mg II column density as seen by the larger quasar beams. We elaborate on this suggestion below. 
In order to demonstrate the effect of beam size on dilution of observed equivalent widths, we have constructed a simple "toy" model. Assume that the Mg II clouds have a clumpy density structure:

$$
\rho(r)= \begin{cases}\rho_{0} & ; r \leq r_{0} \\ \rho_{0}\left(r / r_{0}\right)^{-k} & ; r>r_{0}\end{cases}
$$

Thus, the density of an individual, spherically symmetric absorbing cloud is constant, $\rho_{0}$, within a certain core radius $\mathrm{r}_{0}$, outside of which it drops like a power-law. The maximum column density of a single beam hitting the cloud core is $\mathrm{N}_{M g I I}=\left(2+\frac{2}{k-1}\right) \rho_{0} r_{0}$. On the other hand, if a single beam does not intersect the core, but passes through the cloud at an impact parameter $b$, then it samples a column density $\mathrm{N}_{M g I I} \propto b^{-k+1}$. We calculate the difference in the Mg II column densities produced by such a cloud, absorbing randomly located, uniform brightness beams of different sizes. The column density profile sampled by the beam depends on the size of the beam relative to the core size of the absorber, and how far away from the cloud core the beam passes the absorber, i.e. the impact parameter b. Fig. 1 shows various examples for different scenarios for the specific case of $\mathrm{k}=3.01$ for the powerlaw-slope of the absorber density. In the upper left panel, the beams hits the absorber centrally. Small beams in this case sample much higher column densities than beams larger than the core. In the middle panel, where the centre of the beams just grazes the absorber core, the differences in the column densities seen by the background source become less prominent, but bigger beams still have on average lower average column densities. This situation is reversed for cases where the impact parameter is large enough so that no part of the beams reaches the dense core of the absorber. Not only does the average column density for each beam drop quickly, but now larger beams can sample higher densities due to some parts being much closer to the denser parts of the absorber.

We model the Mg II absorber spectra, superposing Voigt profiles of different column densities $N_{M g I I} / N_{\max }$ and weighing factors $p\left(N_{M g I I}\right)$ taken from the column density distribution functions of figure 1. The lower panels of figure 1 shows the examples computed from the scenarios in the upper panels. The parameters for these profiles were chosen such that a beam sampling an average column density of $0.4 \mathrm{~N}_{\max }=0.4 \times 2.995 \rho_{0} r_{0}$ results in a line of $1.0 \AA$ for a Doppler parameter of $b=80 \mathrm{~km} / \mathrm{s}$. Assuming a core radius $\mathrm{r}_{0}=5 \times 10^{15} \mathrm{~cm}(\mathrm{cf}$.

\footnotetext{
${ }^{1}$ This value of the Doppler parameter reflects roughly both the instrumental resolution of the SDDS spectra, used by P06 in their analysis, and the typical velocity spread of Mg II absorber systems (see e.g. Charlton, J. C. and Churchill, C. W. (1998)). Individual components of such systems have much lower $b$ values (Churchill 2000).
} 
Hao et al. 2007), leads to an estimate for the density of such a cloudlet of $\rho_{0}(\mathrm{Mg}$ II $) \sim 5 \times 10^{-3}$ $\mathrm{cm}^{-3}$. As we stress below, the exact parameters of this "toy model" are not relevant, but are designed to show how geometric "dilution" of observed equivalent width works. The lower left panel of figure 1 shows how different beam sizes and impact parameters affect the strength of the Mg II absorbers. The differences in the equivalent widths for small and large beams are especially pronounced for impact parameters less than $b_{i m p} \sim 0.5 \times r_{0}$ (lower left panel). Notice how slowly moving out to larger $b_{i m p}$ primarily affects the small beams, while the structure of the absorber for the larger beams remains almost unchanged (lower middle panel). At large distances, the reversal in column density sampling mentioned above, finds it equivalent in the larger beams now producing stronger absorbers (lower right panel). By way of calculating such spectra, we can transform the column density distribution into the observable rest-frame equivalent width $W_{\mathrm{r}}$. Fig. 2 shows the probability density of obtaining $W_{\mathrm{r}}(2796 \AA)$ for different beam sizes, again assuming the specific case of $\mathrm{k}=3.01$ for the power-law density coefficient. This probability density is obtained by integrating the impact parameter distribution out to some fiducial value $b_{\max }$ under the assumptions that all impact parameters have the same likelihood, and that there are no other nearby cores within $b_{\max }$. As we stress below, the exact parameters of this "toy model" are not relevant, but are designed to show how geometric "dilution" of observed equivalent width works. As shown in figure 2 , we observe a range of $W_{\mathrm{r}}$ with small beams, but with bigger beams only smaller values of $W_{\mathrm{r}}$ are observed due to dilution effects.

Thus, the higher incidence of GRB sight-lines with strong Mg II absorbers reported by Prochter et al. (P06) follows naturally from the beam dilution for the larger quasar beam. What we have discussed above is a "toy model" designed to explain this observational result. Determination of exact beam sizes of quasars or GRBs, exact core size distribution of Mg II "cloudlets" or their radial density profile is not the intent here. We note, nonetheless, that invoking somewhat different beam sizes and geometry distributions, as well as absorber core size distributions in connection with spatial clustering of multiple such "cloudlets" would change the details of the $W_{\mathrm{r}}$ distribution, but would not change our main results substantially.

This simple models explains one more observational result: Various quasar absorption line studies have revealed that the number of $\mathrm{Mg}$ II absorbers strongly declines with $W_{\mathrm{r}}$. Prochter et al. (2006) find $f\left(W_{\mathrm{r}}\right)=490.37 W_{\mathrm{r}}^{-2.245}$ for $W_{\mathrm{r}}>1 \AA$, where $f\left(W_{\mathrm{r}}\right)$ is the number of systems per unit equivalent width for their SDSS Data Release 3 quasar sample. In our model such a decline follows automatically as producing such systems becomes harder for the larger QSO beams since they always sample regions of low column densities. Again, we point out that reproducing the exact shape of the equivalent width distribution is not our goal here. 


\section{Discussion}

The differences in the Mg II absorption properties of quasars and GRBs can be easily understood if the continuum emitting regions of quasars are several times bigger than those of GRBs. Let us examine whether this assumption is reasonable.

In the standard thin disk model (Shakura \& Sunyaev 1973), the source size for a quasar with an $\mathrm{AB}$ magnitude $m_{A B}$ and an absorber at observed wavelength $\lambda$ is

$$
R \simeq\left(7 \times 10^{15}\right)\left(\frac{c D_{A}}{H_{0}}\right)\left(\frac{\lambda}{\mu \mathrm{m}}\right)^{3 / 2} 10^{-0.2\left(m_{A B}-19\right)} h^{-1} \mathrm{~cm}
$$

On the other hand, the optical afterglow ring radius of a GRB evolves as Waxman 1997; Loeb \& Perna 1998)

$$
R_{s}(t)=4.1 \times 10^{15}\left(\frac{E_{52}}{n_{1}}\right)^{\frac{1}{8}}\left(1+z_{\mathrm{s}}\right)^{-5 / 8}(t / \text { hour })^{5 / 8} \mathrm{~cm}
$$

where the factor involving the source redshift $z_{\mathrm{s}}$ is due to the cosmic time dilation, $E_{52}$ is the "isotropic-equivalent" of the energy release in units of $10^{52} \mathrm{erg} \mathrm{s}^{-1}$ and $n_{1}$ is the ambient gas density in units of $1 \mathrm{~cm}^{-3}$. For the well-observed GRB 060206 at $z_{\text {GRB }}=4.05$, the radius of the afterglow ring is estimated to be $5 \times 10^{15} \mathrm{~cm}$ (Hao et al. 2007) a few hours after the burst, i.e., when their spectra were obtained (see discussion below).

Initial estimates thus imply the GRB emitting regions to be comparable to those of quasars. In reality, however, the GRB size can be significantly smaller than given by the simplistic formula above, if the optical emission from a GRB is dominated by small sections of the potentially visible ring of emission. In many cases the light curve of a GRB afterglow is observed to be very irregular, with significant $(\sim 20 \%)$ deviations from a power-law decay on timescales of 20-30 minutes approximately 1 day after the burst (e.g., GRB 021004: Bersier et al. 2003). Nakar et al. (2003) proposed that such irregular light curves could result from a patchy shell model of the GRB jet, i.e. the jet structure would have strong angular dependence. This would effectively result in a smaller size of the GRB afterglow compared to the numbers above (see also Ioka et al. 2005) - only a part of the narrow ring would dominate the brightness, reducing the size of the GRB beam by a factor of $\sim 10$ down to few $\times \sim 10^{14} \mathrm{~cm}$. We note that the light curve of GRB 060206, for which Hao et al. (2007) have observed strongly variable intervening Mg II and Fe II lines, has a very irregular optical light curve (Stanek et al. 2007).

What do we know about the sizes of AGNs and GRBs from direct observational mea-

surements? An intensive multiwavelength monitoring program of NGC 7469 yielded a time 
delay of 1.5 light days between variations in the optical continuum measured at $6962 \AA$ Collier et al. 1998) relative to those in the ultraviolet continuum measured at $1315 \AA$ (Wanders et al. 1997). This is consistent with the report of Sergeev et al. (2005), where they find evidence for lag times of $\sim 1-20$ light days for the $V, R$, and $I$ bands relative to the $B$ band in 14 nearby $(z<0.1)$ Seyferts. This would imply continuum sizes of order $10^{15}-10^{16} \mathrm{~cm}$ for nearby quasars and sizes that are an order of magnitude or so larger for the more distant, more massive quasars observed in the Mg II studies. On the other hand, the observation of microlensing of lensed quasars is generally less compatible with such large source sizes. The systematic measurement of quasar disk sizes using microlensing is just beginning, but three recent results lead to sizes of $10^{15.0-15.5}, 10^{14.0-14.5}$, and $10^{15.5-16} \mathrm{~h}^{-1} \mathrm{~cm}$ for the gravitational lenses HE0435-1223 (Kochanek et al. 2006), SDSS0924+0219 (Morgan et al. 2006) and Q2237+0305 (Kochanek 2004), respectively, and these scales are broadly consistent with the expectations from thin disk theory. However, other recent quasar microlensing studies claim that optical emission regions of lensed quasars could be much larger than expected from basic disk models by factors of 10-100 (Pooley et al. 2006; Hawkins 2006). Finally, we note that the incidence of $\mathrm{Mg}$ II absorbers along blazar sight lines is also enhanced relative to those of more typical quasars (Stocke \& Rector 1997). If, once again, difference in beam sizes is the cause of this effect, then quasar sizes are bigger than blazar sizes. Kataoka et al. (2000) estimate a blazar beam size of $0.01 \mathrm{pc}$, right in the range of sizes discussed above and so quasar sizes must be larger than $3 \times 10^{16} \mathrm{~cm}$. The only observational constraints on GRB emission region sizes come from the radio scintillations observed in GRB 970508 (Waxman et al. 1998). These observations do not constrain the GRB sizes well, but find that they are broadly consistent with the theoretical expectation. To summarize, our key assumption of quasar optical emission regions being on average several times larger than GRB emission regions is allowed by current constraints on their sizes, both theoretical and from direct observations.

The other key ingredient of our suggestion is that the Mg II absorbers have to be patchy with a characteristic size of $\sim 10^{16} \mathrm{~cm}$. While no direct size measurement of Mg II "clouds" exists, we have some information that is not inconsistent with our proposed, small size. Rauch et al. (2002) studied Mg II absorbers in the three lines of sight towards the lensed quasar Q2237+ 0305 (Huchra's lens) and found that the individual lines of sight do not show the same Mg II cloudlets (see for example their striking Fig. 10, showing a Mg II system at $z=0.827)$. So, the Mg II absorbers are clearly patchy on scales of $<200-300$ pc. Ding et al. (2003), analyzing strong Mg II absorbers towards PG1634+706, find evidence for structures on scales of parsecs to hundreds of parsecs with densities similar to what we derive for our model, and temperatures of $\sim 10,000 \mathrm{~K}$. Interestingly, they point out that there are hints for Mg I pockets of much higher density which could be as small as $\sim 0.001 \mathrm{pc}$, and 
conclude that "they are pervasive in the disk of the Milky Way" and should therefore be seen in absorption through other galaxies. High resolution studies towards globular clusters and binary stars have revealed substantial structure within the Galactic disk ISM down to scales as low as proposed here (e.g. Andrews 2001 and references therein).

It is also of interest that Fischera et al. (2004,2005), analysing the effect of turbulenceinduced clumpiness, arrive at similar column density distribution functions (cf. e.g. Fischera et al. (2004) Fig. 5 with our Figure 1, upper panel). In their model, a physical mechanism (turbulence) creates a clumpy structure in the large-scale structure of a dust screen of galactic scale. Such a 'clumpy screen' then can create the observed difference in the extinction law of dust, and attenuation law seen in the continuum light of galaxies.

We stress again that we are proposing a new idea in which geometric effects account for the differences in absorption incidence in quasar and GRB sightlines. This is not meant to be a rigorous model in which exact sizes of quasars, GRBs or Mg II clouds are calculated. We show, however, that the assumed parameters are reasonable, though the observational or theoretical constraints are not strong. If future observations show that GRB sizes are not smaller than quasars or that Mg II are much bigger, it will clearly invalidate our proposed solution.

Our "toy model" leads to specific observational predictions:

1. GRBs are, by nature, dynamic objects. As a result, our explanation necessarily predicts that the strength and structure of the absorption lines along many GRB sight lines should vary over time as the GRB ring evolves and the beam size changes. This indeed has now been observed in at least one case by Hao et al. (2007) who discovered strongly variable intervening Mg II and Fe II lines at $z=1.48$ in the line of sight to the $z=4.05$ GRB 060206 . 2. Blazars are also extremely variable objects (cf. Stein et al. 1976; Angel \& Stockman 1980), and thus the same effect should be seen along blazar sight lines.

3. We expect the number of weak absorption systems observed in the spectra of GRB to be lower than that for equivalent quasar populations. This follows logically from a number conservation argument. This prediction has not been tested yet due to the observational difficulty of detecting weak features in the transient GRB phenomena and the still rather small number of GRB afterglow spectra.

4. Additionally, as the size of the quasar continuum-emitting region is dependent on the luminosity, a careful study of different luminosity populations of quasars should also reveal a difference in the incidence and strength of intervening absorbers.

5. Micro-lensing of strongly lensed quasars can probe the $\sim 100$ AU scale intergalactic Mg II cloudlets, as proposed by Dong (2006). In short, Mg II lines seen in the spectra of lensed quasars should be seen evolving. 
To summarise, we propose that the difference is due to the larger beam size of quasars relative to GRBs, and the comparable size of Mg II clouds. This leads to specific observational predictions, of which we discuss several. One of our predictions has already been confirmed by Hao et al. (2007). If further confirmed, it will lead to improved constraints on the sizes of quasars, GRBs and Mg II absorbers, as well as confirming our proposed explanation for the puzzling result of P06.

We are grateful for useful suggestions by Sara Ellison and Pat Hall. We also thank the participants of the morning "Astronomy Coffee" at the Department of Astronomy, The Ohio State University, for the daily and lively astro-ph discussion, one of which prompted us to investigate the problem described in this paper. MCB is supported by a Graduate Fellowship of the National Science Foundation.

\section{REFERENCES}

Andrews, S. M., Meyer, D. M. and Lauroesch, J. T. 2001, ApJ, 552, 73

Angel, J. R. P. and Stockman, H. S. 1980, ARA\&A, 18, 321

Bersier, D., et al. 2003, ApJ, 584, L43

Charlton, J. C. and Churchill, C. W. 1998, ApJ, 499, 181

Chen, H.-W., et al. 2005, ApJ, 634, L25

Churchill, C. W. et al. 2000, ApJ, 543, 577

Collier, S. J., et al. 1998, ApJ, 500, 162

Ding, J., et al. 2003, ApJ, 2003, 587

Dong, S. 2006, 2007, ApJ660, 206

Ellison, S. L., Hall, P. B. and Lira, P. 2005, AJ, 130, 1345

Ellison, S. L. , 2006, MNRAS, 372, 38

Fischera, J. and Dopita, M. A. , 2004, ApJ, 611, 919

Fischera, J. and Dopita, M. A. , 2005, ApJ, 619, 340

Hao, H., Stanek, K. Z., Dobrzycki, A., et al., 2007, ApJ, 659, 99 
Ioka, K., Kobayashi, S., \& Zhang, B. 2005, ApJ, 631, 429

Jakobsson, P., et al. 2004, A\&A, 427, 785

Kann, D. A., Klose, S., \& Zeh, A. 2006, ApJ, 641, 993

Kataoka, J., et al. 2000, ApJ, 528, 243

Kochanek, C. S. 2004, ApJ, 605, 58

Kochanek, C. S., et al. 2006, ApJ, 640, 47

Loeb, A., \& Perna, R. 1998, ApJ, 495, 597

Metzger, M. R., et al. 1997, Nature, 387, 878

Morgan, C. W., Kochanek, C. S., Morgan, N. D., \& Falco, E. E. 2006, ApJ, 647, 874

Nakar, E., Piran, T., \& Granot, J. 2003, New Astronomy, 8, 495

Peterson, B. M., et al. 1977, ApJ, 218, 605

Pooley, D., et al. 2007, ApJ, 661, 19

Prochter, G. E., Prochaska, J. X., \& Burles, S. M. 2006, ApJ, 639, 766

Prochter, G. E., et al. (P06) 2006, ApJ, 648, 93

Rauch, M., et al. 2002 ApJ, 576, 45

Sergeev, S. G., et al. 2005, ApJ, 622, 129

Shakura, N. I., \& Sunyaev, R. A. 1973, A\&A, 24, 337

Stanek, K. Z., et al. 2007, ApJ, 654, L21

Stein, W. A. \& Odell, S. L. \& Strittmatter, P. A. 1976, ARA\&A, 14, 173

Stocke, J. T., \& Rector, T. A. 1997, ApJ, 489, L17

Vreeswijk, P. M., Møller, P., \& Fynbo, J. P. U. 2003, A\&A, 409, L5

Vreeswijk, P. M., et al. 2006, A\&A, 447, 145

Wanders, I., et al. 1997, ApJS, 113, 69

Waxman, E. 1997, ApJ, 491, L19 
Waxman, E., Kulkarni, S. R., \& Frail, D. A. 1998, ApJ, 497, 288

York, D. G., et al. 2006, MNRAS, 367, 945 

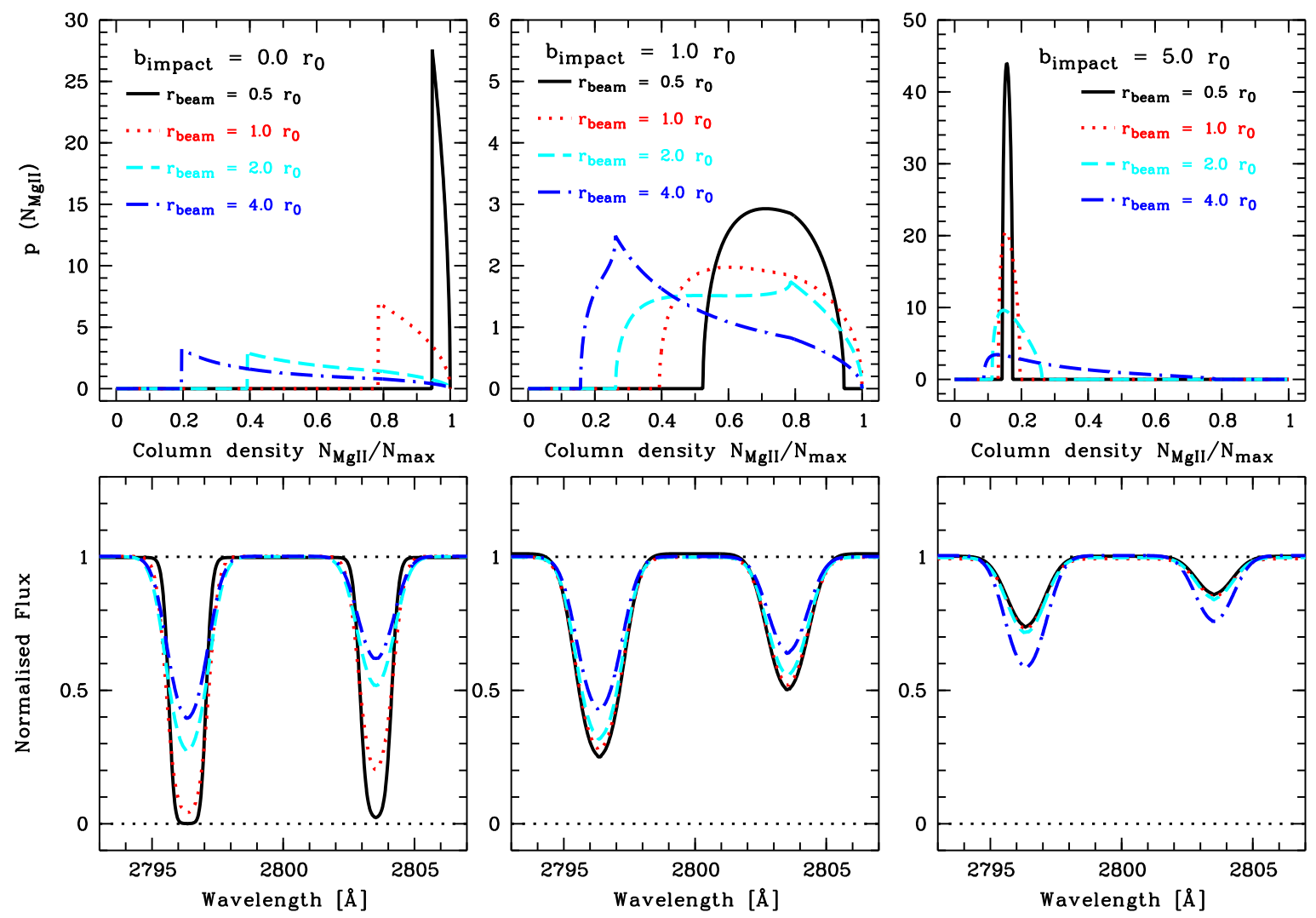

Fig. 1.- Upper panels : The (normalised) column density distribution functions for beams of different size and impact parameters relative to the core size of the absorbers $r_{0}$. These functions have been calculated for the specific case of $k=3$ for the density profile of the absorber. Note how the average column density for low impact parameters (left panel) is a strongly varying function of the beam size. For beams just grazing the dense part of the absorbers (middle panel), the distributions of column densities sampled become more similar, and finally for large impact parameters (right panel), smaller beams sample even lower density regions than their large counterparts. Lower panels : Theoretical spectra computed from the column density distributions above. For the specific parameters (maximum column density $\mathrm{N}_{\max }$, and Doppler parameter $b$ ) of the individual Voigt profiles superposed here, see the text. Note the overall trend to lower equivalent widths from low to high impact parameters (left to right), and how the pronounced differences of the profile depths in the direct hit case (left panel) almost vanish for higher impact parameters (middle and right panels). 


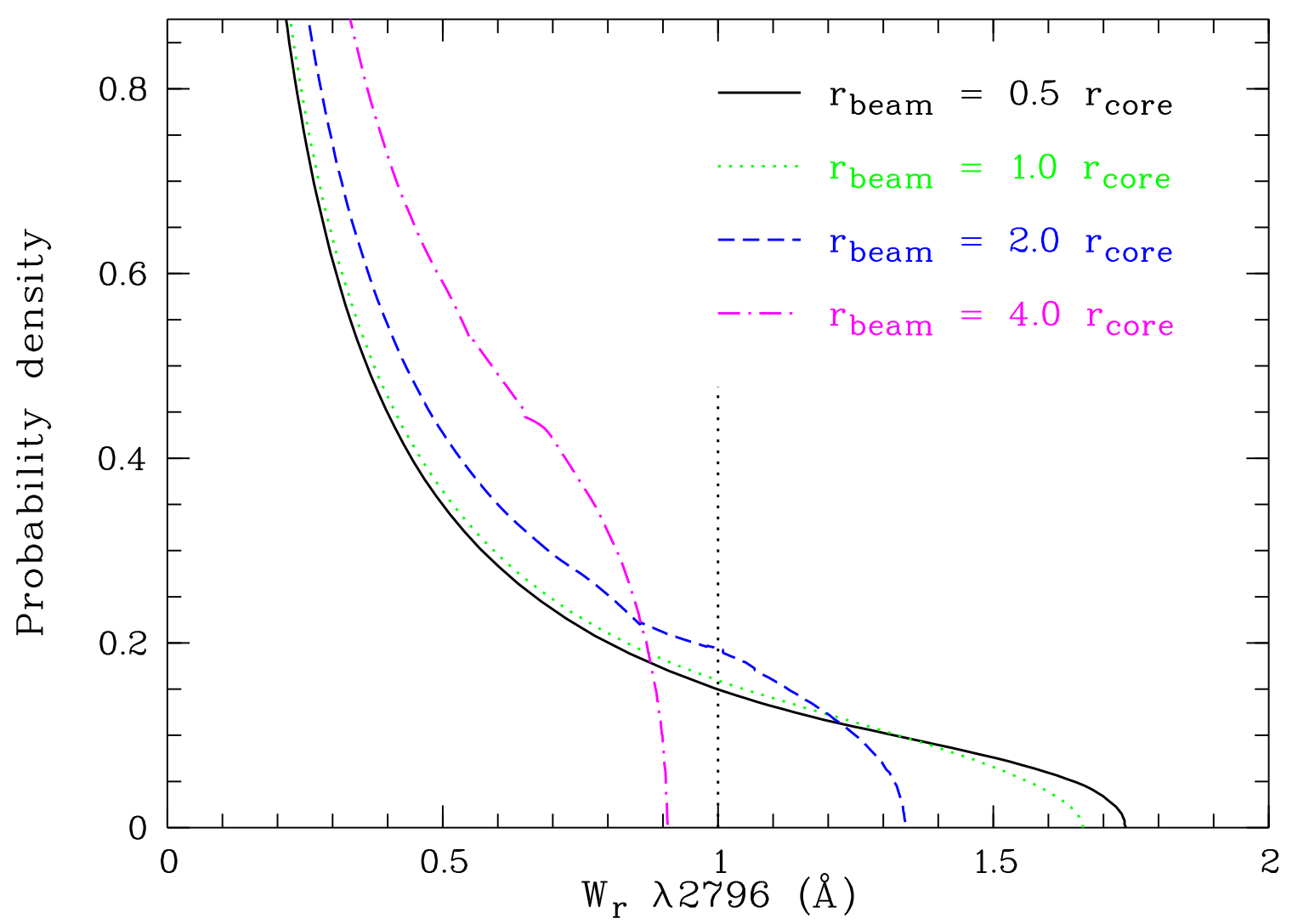

Fig. 2.- The probability density of obtaining $W_{\mathrm{r}}(2796 \AA)$ for beams of various sizes under the assumption of random locations of the cloud core relative to the beams. The transformation between the sampled average column density and $W_{\mathrm{r}}$ was chosen such that an absorber sampling $0.4 \mathrm{~N}_{\max }$ yields a line of $W_{\mathrm{r}}=1.0 \AA$. For details see text. The vertical dotted line indicates the boundary between strong and weak absorbers used by Prochter et al. (P06) for their statistical analysis. Note how the larger beams can only reach low $W_{\mathrm{r}} \mathrm{s}$, readily explaining the scarcity of such absorbers found in quasar studies. The calculations presented here are for the specific case of $\mathrm{k}=3.01$ for the power-law density coefficient. 